\title{
Genome Resource: Ralstonia solanacearum Phylotype II Sequevar 1 (Race 3 Biovar 2) Strain UW848 From the 2020 U.S. Geranium Introduction
}

\author{
Veronica Roman-Reyna, ${ }^{1,2}$ Alicia Truchon, ${ }^{3,4}$ Parul Sharma, ${ }^{5}$ Francesca Peduto Hand, ${ }^{1}$ \\ Reza Mazloom, ${ }^{5}$ Boris A. Vinatzer, ${ }^{6}$ Jonathan M. Jacobs, ${ }^{1,2, \dagger}$ and Caitilyn Allen ${ }^{4, \dagger}$ \\ ${ }^{1}$ Department of Plant Pathology, The Ohio State University, Columbus, $\mathrm{OH}$ \\ ${ }^{2}$ Infectious Disease Institute, The Ohio State University, Columbus, $\mathrm{OH}$ \\ ${ }^{3}$ Microbiology Doctoral Training Program, University of Wisconsin-Madison, Madison, WI \\ ${ }^{4}$ Department of Plant Pathology, University of Wisconsin-Madison, Madison, WI \\ ${ }^{5}$ Department of Computer Science, Virginia Tech, Blacksburg, VA \\ ${ }^{6}$ School of Plant and Environmental Sciences, Virginia Tech, Blacksburg, VA
}

\begin{abstract}
Ralstonia solanacearum phylotype II sequevar 1 (Rsll-1, formerly race 3 biovar 2) causes tomato bacterial wilt, potato brown rot, and Southern wilt of geranium. Strains in Rsll-1 cause wilting in potato and tomato at cooler temperatures than tropical lowland $R$. solanacearum strains. Although periodically introduced, RsIl-1 has not established in the United States. This pathogen is of quarantine concern and listed as a Federal Select Agent. We report a rapidly sequenced ( $<2$ days) draft genome of UW848, a Rsll-1 isolate introduced to the United States in geranium cuttings in spring 2020. UW848 belongs to the near-clonal cluster of Rsll-1 global pandemic strains.
\end{abstract}

\section{Genome Announcement}

Ralstonia solanacearum phylotype II sequevar 1 (Rsll-1, known historically and for regulatory purposes as race 3 biovar 2) causes vascular wilt diseases of diverse ornamental and solanaceaous crops, most notably potato brown rot. Brown rot is among the most serious threats to tropical highland potato growers worldwide. $R$. solanacearum II-1 can be disseminated by latently infected potato seed tubers and geranium cuttings (Scherf et al. 2010; Swanson et al. 2007). Strains in this subgroup, which originated in the Andes but has been globally distributed, induce wilting in plants at cooler temperatures than $R$. solanacearum tropical lowland strains (Allen et al. 2001; Milling et al. 2009). Although the pathogen has been accidentally introduced previously to North America in geranium cuttings, to date it has not become established there. The Rsll-1 subgroup is a quarantine pathogen and a U.S. Select Agent (Lambert 2002). Following an accidental introduction of Rsll-1 in geranium in spring 2020 , we used rapid genome sequencing to identify and characterize the responsible $R$. solanacearum strain.

Bacteria with typical $R$. solanacearum colony morphology were isolated from four wilted geranium plants (cv. Fantasia 'Pink Flare'). All were identified as Ralstonia species based on immunostrip tests (Agdia, Inc., Elkhart, IN) and were determined to be Rsll-1 using the phylotype multiplex PCR and the 630/631 primer pair (Fegan and Prior 2005; Tran et al. 2016). Genomic DNA was extracted from four isolates using Epicentre MasterPure genomic DNA kit and sequenced on an iSeq 100 Illumina benchtop sequencer with the Nextera DNA Flex Library Prep protocol kit and Nextera DNA CD indexes. One hundred fifty bp paired-end

\footnotetext{
${ }^{\dagger}$ Corresponding authors: J. M. Jacobs, jacobs.1080@osu.edu; and C. Allen, callen@ wisc.edu
}

The author(s) declare no conflict of interest.

Accepted for publication 6 July 2020.

\section{Funding}

The authors acknowledge support from the Department of Plant Pathology and C. Wayne Ellet Plant and Pest Diagnostic Clinic at The Ohio State University, Ohio Department of Agriculture Specialty Crops Block Grant (AGR-SCG-19-03) to JMJ, the University of Wisconsin-Madison College of Agricultural and Life Sciences to CA, USDA APHIS (3.0429) to BAV and RM.

\section{Keywords}

Ralstonia solanacearum, geranium, potato, select agent, genomics, diagnostics, race 3 biovar 2 
reads were assembled using SPAdes version 3.13 with k-mer sizes of 55,75,95 (Bankevich et al. 2012). Whole genomes were compared using average nucleotide identity (ANI), with reference genomes from all four phylotypes of the $R$. solanacearum species complex (Remenant et al. 2010; Rodriguez-R and Konstantinidis 2016). Genomes of the four geranium isolates, which were almost identical to each other, had $99.98 \%$ ANI to the well-studied RsIl-1 strain UW551, isolated from geranium in 1999 (Hayes et al. 2017; Williamson et al. 2002).

On average, each genome assembly had 48x coverage and 150 SNPs compared with the UW551 genome ( $\mathrm{Li}$ and Durbin 2009; Li et al. 2009). One selected genome assembly, corresponding to strain UW848, has a GC content of $66.7 \%$, with 5,353,250 bp in 160 contigs. Functional annotation of the contigs was done using Prokka v1.13.3, and secreted proteins were predicted based on SignalP algorithm as part of Prokka analysis (Petersen et al. 2011; Seemann 2014). UW848 genome encodes 4,524 genes, of which 490 are predicted to encode secreted proteins. The temperature-responsive gene cluster lecM-aidA-aidC-solRsoll, required for full virulence, was complete, and the encoded proteins had $100 \%$ amino acid identity with UW551 (Meng et al. 2015). Overall, the genome of Rsll-1 strain UW848 provides a resource for immediate pathogen identification and tracking and for future studies to better understand the molecular epidemiology and biology of this highly regulated quarantine pathogen.

The genome of UW848 is deposited at GenBank under BioSample and Bioproject accession number SAMN15102643 and PRJNA637338, respectively, and was uploaded to LINbase (Tian et al. 2020) where it was assigned the LIN $14_{A} 1_{B} 0_{C} 0_{D} 0_{E} 3_{F} 0_{G} 0_{H} 0_{l} 0_{J} 1_{K} 0_{L} 0_{M} 0_{N} 0_{O} 0_{P} 0_{Q} 3_{R} 0_{S} 0_{T}$.

\section{Literature Cited}

Allen, C., Kelman, A., and French, E. R. 2001. Brown rot. Pages 11-13 in: Compendium of potato Diseases. ed. W. R. Stevenson, R. Loria, G. D. Franc, and D. P. Weingartner, eds. APS Press, St. Paul, MN.

Bankevich, A., Nurk, S., Antipov, D., Gurevich, A. A., Dvorkin, M., Kulikov, A. S., et al, 2012. SPAdes: a new genome assembly algorithm and its applications to singlecell sequencing. J. Comput. Biol. 19:455-477.

Fegan, M., and Prior, P. 2005. How complex is the "Ralstonia solanacearum species complex"? Pages 449-461 in: Bacterial Wilt: The Disease and the Ralstonia solanacearum Species Complex. C. Allen, P. Prior, and A. C. Hayward, eds. APS Press, St. Paul, MN.

Hayes, M. M., MacIntyre, A. M., and Allen, C. 2017. Complete genome sequences of the plant pathogens Ralstonia solanacearum type strain K60 and $R$. solanacearum race 3 biovar 2 strain UW551. Genome Announc. 5: e01088-17.

Lambert, C. 2002. Agricultural Bioterrorism Protection Act of 2002: possession, use and transfer of biological agents and toxins; Interim and Final Rule (7CRF part 331). Fed. Regist. 67:76908-76938.

Li, H., and Durbin, R. 2009. Fast and accurate short read alignment with BurrowsWheeler transform. Bioinformatics 25:1754-1760.

Li, H., Handsaker, B., Wysoker, A., Fennell, T., Ruan, J., Homer, N., et al. 2009. The Sequence Alignment/Map format and SAMtools. Bioinformatics 25:2078-2079.

Meng, F., Babujee, L., Jacobs, J. M., and Allen, C. 2015. Comparative transcriptome analysis reveals cool virulence factors of Ralstonia solanacearum race 3 biovar 2. PLOS One 10:e0139090.

Milling, A., Meng, F., Denny, T. P., and Allen, C. 2009. Interactions with hosts at cool temperatures, not cold tolerance, explain the unique epidemiology of Ralstonia solanacearum race 3 biovar 2. Phytopathology 99:1127-1134.
Petersen, T. N., Brunak, S., von Heijne, G., and Nielsen, H. 2011. SignalP 4.0: discriminating signal peptides from transmembrane regions. Nat. Methods 8 : 785-786.

Remenant, B., Coupat-Goutaland, B., Guidot, A., Cellier, G., Wicker, E., Allen, C., et al. 2010. Genomes of three tomato pathogens within the Ralstonia solanacearum species complex reveal significant evolutionary divergence. BMC Genomics 11:379.

Rodriguez-R, L. M., and Konstantinidis, K. T. 2016. The enveomics collection: a toolbox for specialized analyses of microbial genomes and metagenomes. PeerJ Preprints 4:e1900v1.

Scherf, J. M., Milling, A., and Allen, C. 2010. Moderate temperature fluctuations rapidly reduce the viability of Ralstonia solanacearum race 3 , biovar 2 , in infected geranium, tomato, and potato plants. Appl. Environ. Microbiol. 76: 7061-7067.

Seemann, T. 2014. Prokka: rapid prokaryotic genome annotation. Bioinformatics 30: 2068-2069.

Swanson, J. K., Montes, L., Mejia, L., and Allen, C. 2007. Detection of latent infections of Ralstonia solanacearum race 3 biovar 2 in geranium. Plant Dis. 91: 828-834.

Tian, L., Huang, C., Mazloom, R., Heath, L. S., and Vinatzer, B. A. 2020. LINbase: a web server for genome-based identification of prokaryotes as members of crowdsourced taxa. Nucleic Acids Res. 48:W529-W537.

Tran, T. M., Jacobs, J. M., Huerta, A., Milling, A., Weibel, J., and Allen, C. 2016. Sensitive, secure detection of race 3 biovar 2 and native U.S. strains of Ralstonia solanacearum. Plant Dis. 100:630-639.

Williamson, L., Nakaho, K., Hudelson, B., and Allen, C. 2002. Ralstonia solanacearum race 3 , biovar 2 strains isolated from geranium are pathogenic on potato. Plant Dis. 86:987-991. 